\title{
THE PAYNE EFFECT IN DOUBLE NETWORK ELASTOMERS
}

\author{
J. WANG \\ Chemistry Department, George Mason University \\ FAIRFAX, VA 22030 \\ G. R. HAMED, * K. UMETSU \\ Institute of Polymer Science, The University of AKron \\ AKRON, OH 44325-3909 \\ C. M. RoLAND** \\ Chemistry Division, Naval Research Laboratory \\ WASHINGTON, D.C. 20375-5342
}

\begin{abstract}
Double network elastomers were prepared by curing under strain previously-crosslinked natural rubber or styrenebutadiene copolymer. The rubbers were reinforced with carbon black, so that the conventional (singly-cured) materials exhibited a substantial Payne effect, reflecting agglomeration of the filler particles. This effect was much reduced in the double networks - the storage modulus varied more weakly with strain amplitude, and the mechanical hysteresis was substantially smaller. Comparable results were obtained for dynamic mechanical measurements employing different test geometries; that is, the effect is independent of the direction of the strain relative to the orientation of the double network. These results indicate that deformation during the imposition of a second network disrupts the carbon black agglomerates, and this deflocculated structure is stabilized by the second crosslinking. Thus, double network processing is a general means to lower the hysteresis of filled rubbers.
\end{abstract}

\section{INTRODUCTION}

Orientation has a significant effect on the mechanical response of elastomers, especially rubbers which strain-crystallize. Although this orientation is usually not stable in a flexible chain polymer above its glass transition temperature, permanent orientation can be achieved via a "double network". After an initial, isotropic crosslinking, the rubber is stretched and crosslinked a second time. While this process happens inadvertently, with the consequence referred to as permanent set, ${ }^{1-3}$ double networks can also be obtained by design. Control of the orientation and crosslink apportionment yields higher modulus, ${ }^{4-6}$ enabling the usual compromise between elastomer stiffness and strength to be circumvented. For strain-crystallizing rubbers, the benefits are even greater, since substantial improvements in crack-growth and fatigue performance can be realized. ${ }^{7-10}$ Double networks exhibit anisotropic properties, ${ }^{7-9,11,12}$ and are usually characterized in terms of their residual stretch ratio, $\lambda_{\mathrm{R}}$, equal to the ratio of their length along the stretch direction of the second cure to the initial length (prior to the second curing, or equivalently, in the uncured state). However, $\lambda_{\mathrm{R}}$ does not uniquely define a double network, as different cure strains and crosslink apportionment between the two networks can yield the same residual strain, but different mechanical properties.

Most studies of double networks utilize unfilled rubbers, in order to better understand structure-property relationships. In an elastomer containing reinforcing filler such as carbon black or silica, agglomeration of the particles produces a filler network, in addition to the network of covalently-bonded polymer chains. In fact, Reichert et al. ${ }^{13}$ modeled the deformation of conventional (single) networks of filled rubber as a double network, adopting an approach similar to that used to analyze unfilled double networks. ${ }^{1,14-16}$ This implies that double networks reinforced

* Corresponding author. Ph: 330-972-6831; Fax: 330-972-5290; email: hamed@polymer.uakron.edu

** Corresponding author. Ph: 202-767-1719; Fax: 202-767-0594; email: roland@nrl.navy.mil 
with filler can be viewed as a composite of three distinct networks. Roland and Peng ${ }^{17}$ investigated the electrical properties of carbon black filled double networks, finding that the electrical conductivity was lower than for single networks. After an initial minimum at low strains, however, the conductivity of the double networks increased more strongly with strain. Hamed and Huang ${ }^{9}$ observed highly anisotropic mechanical properties in natural rubber double networks containing carbon black, the tensile and tear strengths being higher for deformations parallel to the orientation. These double networks were especially "notch sensitive", with substantial reductions in strength accompanying the introduction of small cuts.

Practical utilization of double networks for most applications would require the use of carbon black or other fillers, given the performance and cost benefits. ${ }^{18}$ However, there is a wellknown drawback to the use of reinforcing fillers - higher mechanical hysteresis due to agglomeration of the filler particles. This agglomerate structure, which underlies the Payne effect, ${ }^{19}$ reduces the mechanical efficiency of rubber and contributes to heat build up. For a typical rubber compound, roughly half of the energy dissipation during cyclic deformation can be ascribed to the agglomerated filler, the rest coming from chain ends and internal friction..$^{20}$ Empirical relations have been derived which relate the propensity of carbon black to agglomerate with the heat buildup of the rubber compound. Minimizing this hysteresis can be a major criterion in developing a rubber compound. ${ }^{21}$ For example, tire rolling resistance is the second largest source of fuel consumption in an automobile, and rubber hysteresis is the primary energy loss mechanism of a rolling tire. ${ }^{22,23}$ Thus, means to reduce filler agglomeration are of obvious value.

As illustrated by the Payne effect, ${ }^{18,19}$ deformation disrupts the agglomerate structure, increasing the resiliency of rubber. Thus, intensive mixing of a rubber compound to disperse and distribute the filler aggregates is a key aspect to minimizing the agglomeration and obtaining good mechanical properties. Since imposition of a large strain is inherent to producing double networks, potentially they are a route to lower hysteresis rubber. Substantial filler flocculation also transpires after mixing. ${ }^{24,25}$ However, the crosslinking of a double network after straining may stabilize the de-agglomerated filler network. Consistent with this idea is the earlier finding of lower electric conductivity in filled double networks. ${ }^{17}$ The flocculated carbon black is the primary conductive pathway, so lower conductivity suggests less agglomeration. In the present study, the mechanical hysteresis of carbon black filled double networks of polyisoprene was investigated. Dynamic moduli were measured using different sample geometries, giving different deformations relative to the double network orientation. The results indicate substantial reduction in filler agglomeration, and thus improved mechanical performance.

\section{EXPERIMENTAL}

Two cis-1,4-polyisoprene rubber formulations were used (Table I). For the natural rubber (NR) samples, the initial crosslinking was carried out in a closed mold at $135{ }^{\circ} \mathrm{C}$ for $24 \mathrm{~min}$. The rubber was then elongated, and held in a frame contained between two aluminum plates. The stretch ratio, $\lambda_{c}$, was determined from fiducial marks. The second curing was carried out in vacuo, with heat applied via electric heaters embedded in the two aluminum plates, whose temperature was independently controlled. A temperature of $170{ }^{\circ} \mathrm{C}$ was attained in $20 \mathrm{~min}$, and then maintained for $16 \mathrm{~min}$, followed by quenching. 
TABLE I

FORMULATIONS

\begin{tabular}{lll}
\hline & NR & IR \\
\hline Deproteinized natural rubber $^{\mathrm{a})}$ & 100 & - \\
\hline Synthetic natural rubber ${ }^{\mathrm{b}}$ ) & - & 100 \\
\hline $\mathrm{N} 234$ & 50 & - \\
\hline $\mathrm{N} 330$ & - & 40 \\
\hline Dicumyl peroxide & 2 & - \\
\hline Stearic acid & - & 2 \\
\hline Zinc oxide & - & 3 \\
\hline N-isopropyl-N-phenyl-p-phenylenediamine & - & 2 \\
\hline 4,4-dithiodimorpholine & - & 1 \\
\hline N-cyclohexyl-2-benzothiazole sulfonamide & - & 1 \\
\hline
\end{tabular}

a) DPNR-S from the H.A. Astlett Co.

b) Nipol IR-2200 from Zeon Chemicals.

For shear testing, rings (inner and outer diameters equal to 19 and $25 \mathrm{~mm}$, respectively) were cut from the cured sheets, and adhered to parallel steel plates. Sample thicknesses varied from 2.5 to $3.8 \mathrm{~mm}$, depending on the residual stretch ratios, $\lambda_{\mathrm{R}}$. The measurements were done at $30{ }^{\circ} \mathrm{C}$ and $10 \mathrm{~Hz}$, using a TA Instruments ARES-LS.

Dynamic measurements in tension were carried out using a Dynastat instrument. Samples ( $24 \mathrm{~mm}$ long and $3.7 \mathrm{~mm}$ wide) were cut with the long axis parallel to the stretch direction of the double network. A static offset equal to $115( \pm 10) \%$ of the dynamic amplitude was used to avoid buckling. The measurements were done at RT and $0.1 \mathrm{~Hz}$.

Crosslink densities (Table II) were estimated from the equilibrium modulus, using the rubber elasticity equation for affine deformations. ${ }^{26}$ Since the orientation of a double network influences its modulus, all determinations were done on samples cured in the isotropic state. The assumption was made that stretching does not affect the crosslinking reactivity, which is reasonable for rubbery materials.

TABLE II

DOUBLE NETWORKS

\begin{tabular}{lccc}
\hline & $v_{2} / v_{1}$ & $\lambda c$ & $\lambda_{\mathrm{r}}$ \\
\hline NR-D1 & 3.4 & 1.41 & 1.34 \\
\hline NR-D2 & 3.4 & 1.82 & 1.67 \\
\hline NR-D3 & 3.4 & 2.16 & 2.00 \\
\hline NR-D4 & 3.4 & 2.69 & 2.40 \\
\hline IR-D1 & 1.2 & 1.50 & 1.15 \\
\hline IR-D2 & 3.1 & 1.50 & 1.30
\end{tabular}

$v_{2} \equiv$ crosslink density of second network; $v_{1} \equiv$ crosslink density of first network.

\section{RESULTS}

The residual strain of double networks is a function of both the cure strain and the crosslinking apportionment. This is demonstrated in Figure 1, in which the residual stretch ratio is shown for double networks having a fixed crosslinking apportionment, and for double networks cured 
at a fixed value of strain. There is proportionality between $\lambda_{R}$ and $\lambda_{c}$, while $\lambda_{R}$ varies sublinearly with crosslink apportionment. Although a double network is not uniquely defined by the residual strain, its optical and mechanical properties are generally governed by the magnitude of $\lambda_{R}$. Whether this holds true for the low strain dynamic properties of filled rubbers is examined below.

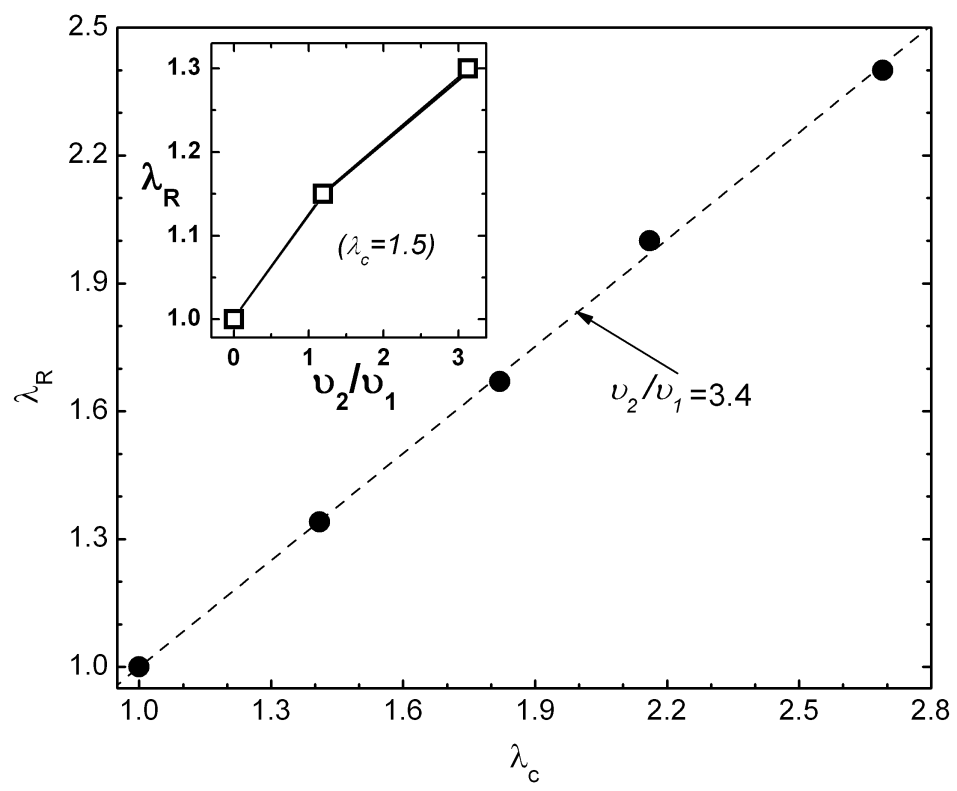

FIG. 1. - Residual stretch ratio as a function of the stretch ratio during the second crosslinking, for NR double networks having $77 \%$ of their total crosslinks in the second network. The inset shows $\lambda_{\mathrm{R}}$ versus the ratio of crosslinks in the two networks, for IR double networks prepared at a cure strain of $50 \%$.

\section{PEROXIDE-CROSSLINKED DEPROTEINIZED NATURAL RUBBER}

Figure 2 shows the dynamic storage, G', and loss, G', moduli measured in shear for two double networks having respective $\lambda_{R}=1.34,1.67$ and 2.00, along with data for a single network $\left(\lambda_{R}=1\right)$. For the latter, which experienced identical processing and heat history, there is a plateau in G', reflecting the carbon black network. At a strain amplitude on the order of $0.2 \%$, the storage modulus begins to decrease, as deformation breaks up the flocculated filler particles. This breakup is associated with a maximum in the loss modulus, centered around $1 \%$ strain. The behavior of the double networks is qualitatively similar, but the effect is weaker. The plateau in G' and the maximum in G' are both $c a$. one-third lower. These results show that the carbon black agglomeration is reduced in the double networks. However, the magnitude of this reduction does not depend on the residual strain of the double network; any differences in the degree of filler deagglomeration are within the experimental scatter. 


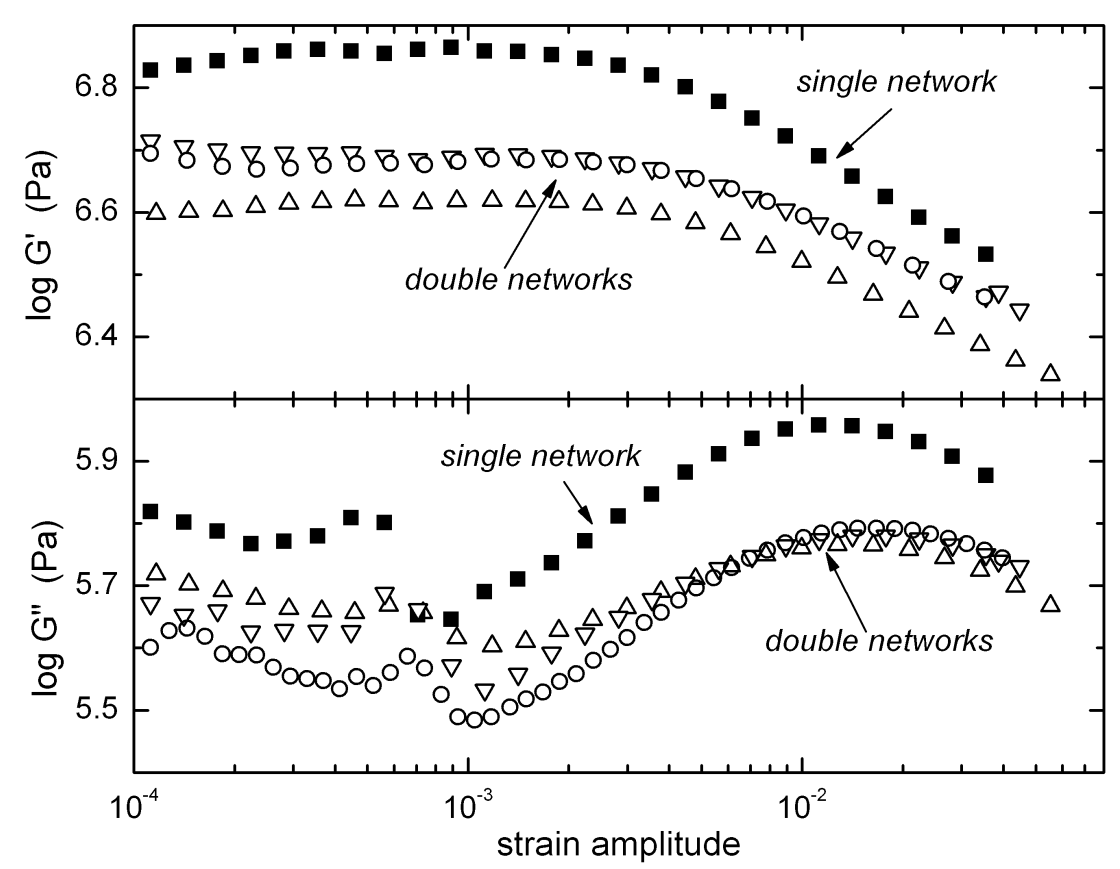

FIG. 2. - Dynamic storage (upper panel) and loss (lower panel) moduli of single network (filled symbols) and double networks (NR-D1 $\triangle$; NR-D2 $\bigcirc$; NR-D3 $\nabla$ ), measured using torsional shear of ring samples at $10 \mathrm{~Hz}$ and $30{ }^{\circ} \mathrm{C}$. The plateau in $\mathrm{G}^{\prime}$ is due to flocculated filler, the disruption of which at higher strain gives rise to the maximum in G". The magnitude of these two characteristic features is smaller, reflecting less carbon black agglomeration, in the double networks. The structure in the loss moduli data below ca. $0.1 \%$ strain is an instrumental artifact.

The data in Figure 2 were obtained on ring samples, twisted (sheared) in the plane of the double network orientation. Generally, the mechanical properties of double networks exhibit some anisotropy, ${ }^{7-9,11,12}$ so it is of interest to assess the Payne effect for other deformation modes. In Figure 3 are results for tension, with the principle axis parallel to the stretching during cure. The range of the data is more restricted than in Figure 2, due to instrumental limitations; nevertheless, there is a clear reduction in both the storage and loss moduli for the double network. In both Figures 2 and 3, the dynamic strain associated with breakup of the filler network is not affected by the presence of the double network.

\section{SULFUR-VULCANIZED SYNTHETIC POLYISOPRENE}

To demonstrate that the effects describe above are not unique to the peroxide-cured NR, sulfur-cured double networks were produced using synthetic cis-1,4-polyisoprene (IR-1 and IR-2 in Table II). The measurements were also carried out differently, via torsion of a rectangular strip. This deformation involves bending as well as shear modes perpendicular to the double network principle orientation. The storage moduli for the two double networks and a (control) single network are shown in Figure 4. In comparison to the isotropic sample, the double networks exhibit a marked reduction $(\sim 40 \%)$ in the plateau associated with the carbon black network. Thus, different elastomers, tested using a different test geometry, corroborate the prior results that the Payne effect is reduced for double networks. Note that unlike the results in Figure 3, the double network having a larger residual strain appears to have a weaker Payne effect. This reflects trivial variations in the preparation and processing of the materials; overall, no consistent trend with $\lambda_{\mathrm{R}}$ was observed. 


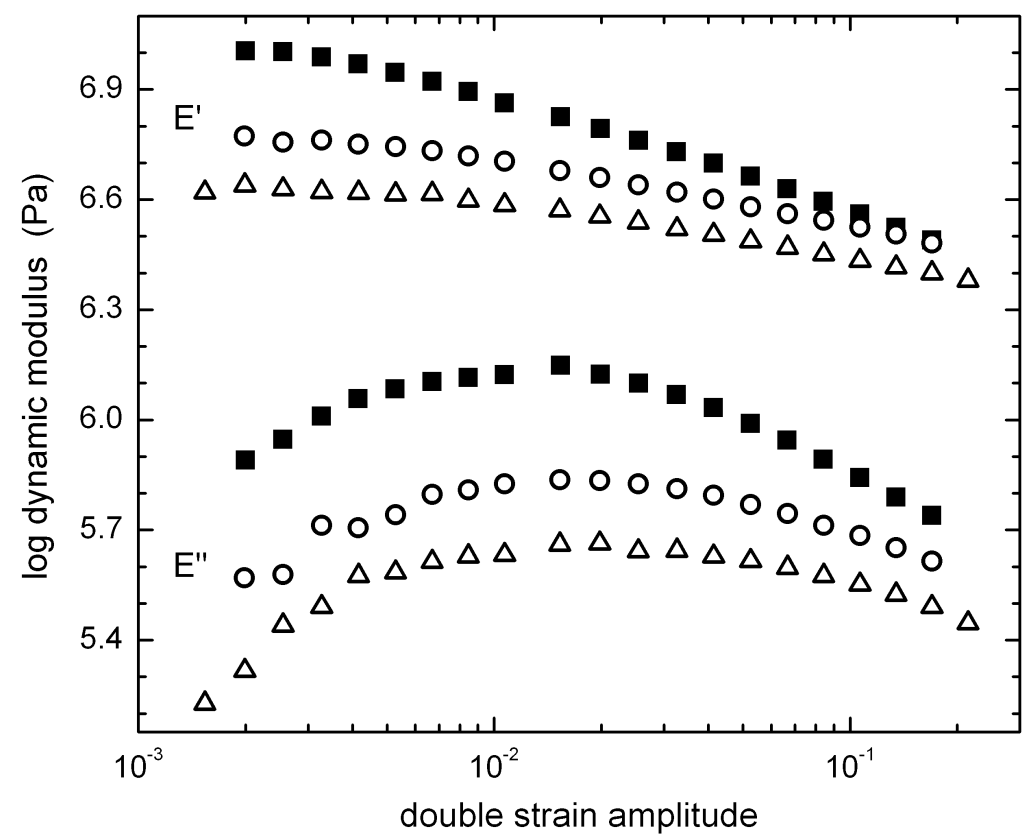

FIG. 3. - Dynamic storage (upper curves) and loss (lower curves) moduli of two double networks $(\mathrm{NR}-\mathrm{D} 2 \bigcirc$; NR-D4 $\triangle$ ) and the corresponding single network $(\mathbf{\square})$, measured in tension, applied parallel to the orientation of the double network at $0.1 \mathrm{~Hz}$ and RT.

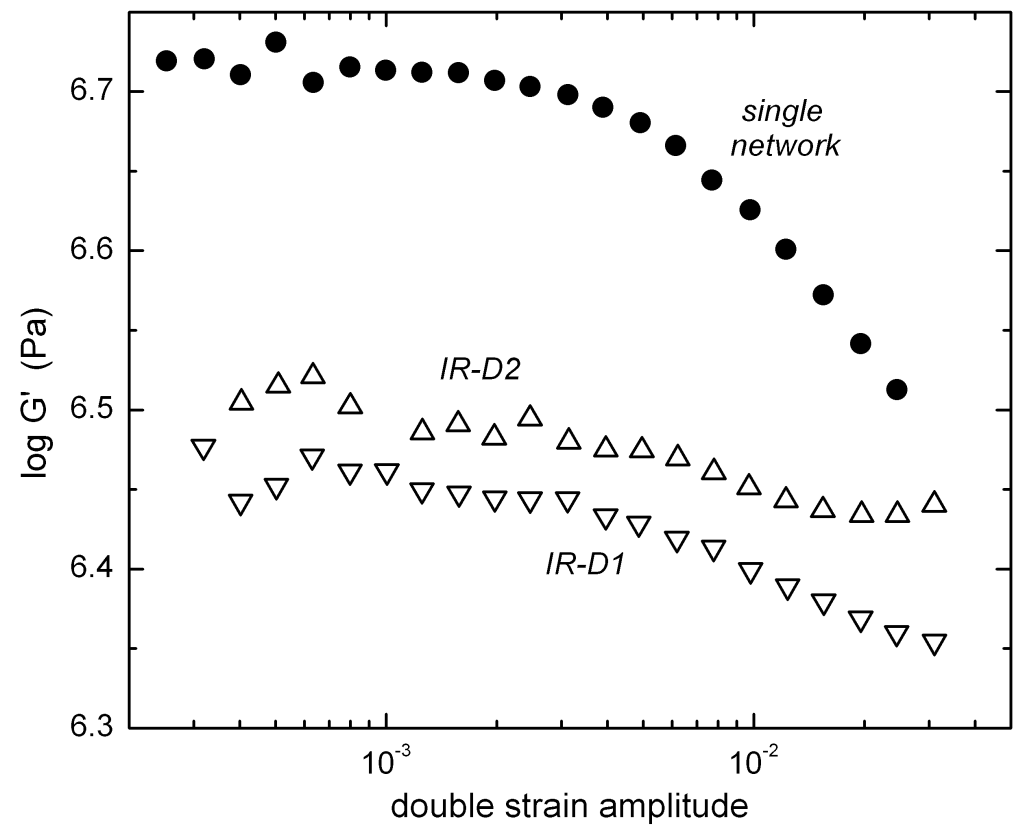

FIG. 4. - Dynamic storage modulus of double networks (hollow symbols) and the corresponding single network (filled symbols), measured using torsional shear of rectangular strips at $1 \mathrm{~Hz}$ and RT. The absence of a filler network in the double networks occasions the disappearance of the strain-dependent plateau. 


\section{CONCLUSIONS}

The mechanical inefficiency and heat buildup associated with agglomerated carbon black is a well-known problem in rubber technology. When a double network is produced, the stretching during the second cure disrupts the agglomerated filler network, since the strain, as well as the residual strain, is always much greater than the strain $(\sim 1 \%)$ associated with disruption of the filler network. The deflocculated structure is stabilized by the second crosslinking, leading, as shown herein, to rubber with substantially reduced mechanical hysteresis. The fact that elongation was used to produce the double networks means that there is orientation along this direction in the final material. Consequently, certain mechanical and failure properties exhibit anisotropy. However, the lower dynamic hysteresis observed herein is independent of the deformation direction. The only requirement is that the connectivity of the flocculated filler structure be disrupted prior to the second cure. This lack of any orientation effect is demonstrated herein by dynamic measurements using three different geometries - shear (in the plane of the double network principle orientation), uniaxial extension (parallel to the orientation of the double network), and torsion (combining bending and shear deformations). For all cases, the double networks showed lower hysteresis. Thus, the incorporation of a double network structure may be a generally useful approach to more energy efficient elastomers.

\section{ACKNOWLEDGEMENTS}

The work at NRL was supported by the Office of Naval Research, and that at the University of Akron by the D'Ianni Research Endowment and a consortium of companies supporting the Rubber Research Center.

\section{REFERENCES}

${ }^{1}$ R. D. Andrews, A. V. Tobolsky and E. E. Hanson, J. Appl. Phys. 17, 352 (1946).

${ }^{2}$ J. Scanlon and W. F. Watson, Trans. Farady Soc. 54, 740 (1958).

${ }^{3}$ K. T. Gillen, Macromolecules 21, 442 (1988).

${ }^{4}$ K. J. Smith and R. J. Gaylord, J. Polym. Sci. A-2 10, 283 (1972).

${ }^{5}$ C. M. Roland and M. L. Warzel, Rubber Chem. TeChnol. 63, 285 (1990).

${ }^{6}$ P. G. Santangelo and C. M. Roland, Rubber Chem. Technol. 67, 359 (1994).

${ }^{7}$ P. G. Santangelo and C. M. Roland, Rubber Chem. Technol. 68, 124 (1995).

${ }^{8}$ S. Kaang and C. Nah, Polymer 39, 2209 (1998).

${ }^{9}$ G. R. Hamed and M. Y. Huang, Rubber ChEM. TeChnOL. 71, 846 (1998).

${ }^{10}$ P. G. Santangelo and C. M. Roland, Rubber Chem. TeChnol. 76, 892 (2003).

${ }^{11}$ A. Greene, K. J. Smith and A. Ciferri, Trans. Farady Soc. 61, 2772 (1965).

${ }^{12}$ P. H. Mott and C. M. Roland, Macromolecules 33, 4132 (2000).

${ }^{13}$ W. F. Reichert, D. Goritz and E.J. Duschl, Polymer 34, 1216 (1993).

${ }^{14}$ P. J. Flory, Trans. Farady Soc. 56, 722 (1960).

${ }^{15}$ L.G. Baxandall, and S.F. Edwards, Macromolecules 21, 1763 (1988).

${ }^{16}$ T. Termonia, Macromolecules 23, 1976 (1990).

${ }^{17}$ C. M. Roland and K. L. Peng, Rubber Chem. Technol. 64, 790 (1991).

${ }^{18}$ A. I. Medalia and G. Kraus in "Science and Technology of Rubber," J. E. Mark, B. Erman, and F.R. Eirich, Eds., Academic Press, New York, 1994, ch. 8.

${ }^{19}$ A. R. Payne, J. Appl. Polym. Sci. 7, 873 (1963).

${ }^{20}$ J. D. Ulmer, W. L. Hergenrother and D.F. Lawson, RubBer Chem. TechnOL. 71, 637 (1998).

${ }^{21}$ F. S. Conant, Rubber Chem. Technol. 48, 397 (1975). 
${ }^{22}$ D. J. Schuring and S. Futamura, RubBer Chem. TeChnOl. 63, 315 (1990).

${ }^{23}$ D. E. Hall and J. C. Moreland, Rubber Chem. Technol. 74, 525 (2001).

${ }^{24}$ D. Bulgin, Trans. Inst. Rubber Ind. 21, 188 (1945).

${ }^{25}$ G. G. A. Bohm and M. N. Nguyen, J. Appl. Polym. Sci. 5, 1041 (1995).

${ }^{26}$ P. H. Mott and C. M. Roland, Macromolecules 29, 6941 (1996).

[ Received June 2004, revised January 2005 ] 